This item was submitted to Loughborough's Research Repository by the author.

Items in Figshare are protected by copyright, with all rights reserved, unless otherwise indicated.

\title{
Understanding the contribution of operator measurement variability within flow cytometry data analysis for quality control of cell and gene therapy manufacturing
}

\section{PLEASE CITE THE PUBLISHED VERSION}

https://doi.org/10.1016/j.measurement.2019.106998

\section{PUBLISHER}

Elsevier

\section{VERSION}

AM (Accepted Manuscript)

\section{PUBLISHER STATEMENT}

This paper was accepted for publication in the journal Measurement and the definitive published version is available at https://doi.org/10.1016/j.measurement.2019.106998.

\section{LICENCE}

CC BY-NC-ND 4.0

\section{REPOSITORY RECORD}

Grant, Becky, Karen Coopman, Nick Medcalf, Sandro Silva-Gomes, Jonathan Campbell, Bo Kara, Julian Braybrook, and Jon Petzing. 2019. "Understanding the Contribution of Operator Measurement Variability Within Flow Cytometry Data Analysis for Quality Control of Cell and Gene Therapy Manufacturing". Loughborough University. https://hdl.handle.net/2134/9742451.v1. 
UNDERSTANDING THE CONTRIBUTION OF OPERATOR MEASUREMENT

VARIABILITY WITHIN FLOW CYTOMETRY DATA ANALYSIS FOR QUALITY CONTROL OF CELL AND GENE THERAPY MANUFACTURING

9

Rebecca Grant ${ }^{1}$, Karen Coopman ${ }^{1}$, Nicholas Medcalf ${ }^{1}$, Sandro Silva-Gomes ${ }^{2}$, Jonathan J. Campbell $^{3}$, Bo Kara², Julian Braybrook ${ }^{3}$, Jon Petzing ${ }^{1}$.

1. Loughborough University, Loughborough, Leicestershire, LE11 3TU, UK

2. GlaxoSmithKline R\&D, Cell \& Gene Therapy, Stevenage, Hertfordshire, SG1 2NY,

Corresponding Author:

Rebecca Grant,

23 Wolfson School of Mechanical, Electrical and Manufacturing Engineering, Loughborough

24 University, Loughborough, Leicestershire, LE11 3TU, UK.

25 E-mail: r.grant@lboro.ac.uk 


\section{Abstract - 150 words}

Flow Cytometry is a measurement technique used in Quality Control and in-process measurements of biomanufactured Cell and Gene Therapy products. However, it contains a number of sources of measurement variation at; sample preparation, instrument setup, analysis, and post-analytical data analysis stages. The latter sees variation introduced from operator subjectivity, which is investigated here to understand what effects the interpretation of diagrammatical protocols have on inter-operator analysis.

36 operators from different sites were given a series of histograms to analyse, gating a shifting peak. This was repeated with diagrammatical protocols to apply gates which reduced inter-operator variation by up to $92 \%$. Various control limits include and exclude different results and when adjusted with a log transform differences in outlier discrimination have been found. This research supports the use of Flow Cytometry diagrammatical protocols to reduce the contribution of inter-operator variation and measurement uncertainty associated within Cell and Gene Therapy manufacturing scenarios.

\section{Keywords}

Operator Variation, Cell Therapy Manufacture, Quality Control, Data Analysis, Flow

$$
\text { Cytometry, Biometrology }
$$

\section{Highlights}

- There is up to a $92 \%$ reduction in variation when operators follow prescribed protocols for analysis.

- Different control limits can be used to define in or out of specification results, but the effect of these limits must be carefully chosen to ensure values falling out of bounds are understood.

- Log transforms, when applied to 'normalise' data, can change the outliers defined by control limits. 
0

\subsection{Introduction}

High value products require significant attention during their manufacture, to ensure consistency of product to a detailed, quality release specification. High-value manufacture covers a number of different industries, with Cell and Gene Therapy (CGT) manufacturing [1] being a rapidly growing sector within the UK. The market for CGT products has grown significantly in potential in recent years, and with licensed products such as Yescarta ${ }^{\text {TM }}$ [2] and Kymriah ${ }^{\mathrm{TM}}$ [3] becoming available, an ever-increasing focus must be paid to Quality Control (QC) of products, to maintain high standards now, and when scale up or out across distributed biomanufacturing platforms becomes a possibility.

Any deviation in the biomanufacturing, and uncertainty in the measurement, can waste critical time and costly materials, thereby impacting the safety of the patient as well as increasing the cost of goods. Improved control over biomanufacture and release provides greater certainty in decision-making, allowing better success rates of the expensive therapeutics through the biomanufacturing route, thus better delivery of high-quality therapeutics to the patient, although it is noted that measurement uncertainty may well persist and requires management.

1 Flow Cytometry (FC) is a significant Quality Control $\left(\mathrm{QC}^{1}\right)$ release measurement technique used to test metrics such as cell count, purity and product characteristics attributed to cellular phenotype and product definition [4]. FC is a laser-based analytical method often using up to 18 exclusive analysis channels, that can interrogate multiple combinations of fluorescent biomarkers (used to uniquely tag cells) at the single cell level through the use of specific antibodies conjugated to distinct fluorophores. Cells can be

\footnotetext{
${ }^{1} \mathrm{QC}=$ Quality Control
} 
labelled with specific antigen markers which help to identify specific cellular states with positive and negative controls. A preparation of labelled cells are hydrodynamically focused through a laser pathway, with fluorescence emission spectra from each fluorophore being recorded, using narrow line width optical separation mirrors and filters to allow sensors and detectors to pick up specific bandwidths of light emitted from the fluorophore markers attached to the cell surface.

Sample preparation time is lengthy and the irreversible nature of tagging the cells for this technique currently limits its use in-process for Cell Therapy Product (CTP) ${ }^{2}$ development, where regulations stipulate that a sample cannot be administered to a patient postanalysis. FC instruments can be integrated into Fluorescence Activated Cell Sorting $(\mathrm{FACS})^{3}$ platforms to sort the required cells on the basis of electrical impedance, but the principle remains the same and the irreversible nature of fluorescence tagging means these samples still cannot be administered post-analysis. Variation in staining reagents and the sample preparation itself is a well-known problem in the industry, with many international efforts made to reduce variation from these sources [6-11].

To set up the FC system, voltages relating to sensor gain and amplification of fluorescence signals are set for the relevant laser channel being used for excitation of specific markers. This can be optimised with fluorescent calibration beads and antigen marker titration, to ensure cellular populations can be fully visualised and are not close to limits of sensitivity of the instrument. The data relating to the measured fluorescence is visualised using a series of scatter plots, with each point representing a single measured event or cell [5]. Typical FC analysis of cells requires a minimum of 30,000 cell events to be acquired.

\footnotetext{
${ }^{2}$ CTP $=$ Cell Therapy Product

${ }^{3}$ FACS $=$ Fluorescence Activated Cell Sorting
} 
101 These cell events can be visualised as histograms (for just one marker), or scatter plots to

102 visualise two or more markers at the same time. Different positive and negative

103 fluorescence areas can help to identify positive and negative markers on the cell surface

104 which relate to different cellular states to identify.

105

Assay or test determination and discrimination is a function of interpretation of the FC output data, by an operator often applying a series of subjective software gates within multi-dimensional scatter plots to determine positive and negative thresholds. This requires correct discrimination of data sub-populations that are characterised by noisy clusters of data, which introduces inherent subjectivity and increased measurement uncertainty in the data analysis and assay conclusions.

$\mathrm{FC}$ is an example of complex metrology whereby whole instrument uncertainty statements have yet to be fully defined. There are many sources of variation which can contribute to the overall uncertainty of FC measurements, with the Ishikawa diagram in Figure 1 illustrating many of these components. The focus of the research reported here is, for the first time, to understand the impact of operator subjectivity within Flow Cytometry data analysis for a QC Cell Therapy exemplar. With respect to Figure 1, this is the Operator branch of the Ishikawa diagram and encompasses all elements apart from the parallax term. In addition, for the first time this research starts to define the variation magnitude that

121 would help to quantify an operator uncertainty term. In addition, common statistical tools are mapped to determine the consequence on outlier definition and exclusion. Whilst the work reported is not intended to develop or deliver a whole instrument uncertainty statement, it is fully anticipated that the results of the operator variation analysis will eventually inform and contribute to a detailed uncertainty analysis. 


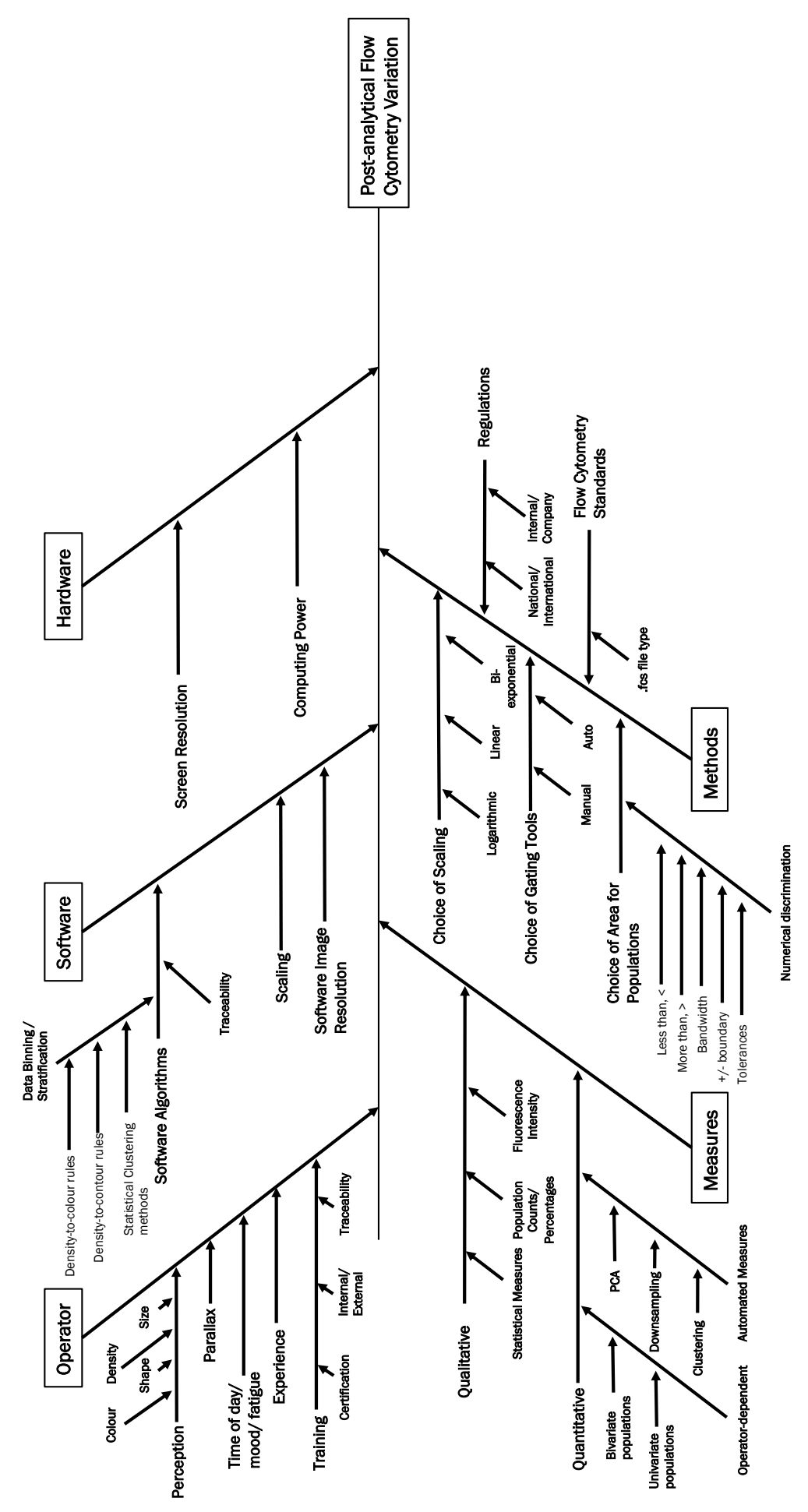

129 Figure 1: Ishikawa diagram listing multiple sources of uncertainty

$130 \quad 2$ column width 
132 This paper has been organised to describe the protocols and standards used to generate 133 files for subjectivity studies (Section 2), with a definition of the statistical tools and outlier 134 discriminators used for data processing defined in Section 3, whilst Sections 4 and 5 135 demonstrate how these studies were conducted with participants. The study results have 136 been discussed in Section 6, with greater emphasis paid to outlier boundary estimators in 137 Section 7, to identify differences in control limits used within manufacturing contexts. 


\subsection{Flow Cytometry Protocols and Standards}

\subsection{Standards and regulation}

In any manufacturing context, it is important to know and quantify the sources of variation in a process or measurement, to better comprehend the allowable fluctuation within a system. Within biomanufacturing and its associated regulatory capacity, all clinical testing or pathology laboratories complying to ISO 15189 guidelines need to be able to quantify uncertainty within a process or measurement [12] and are supported by the International Committee for Harmonization $(\mathrm{ICH})^{4}$ Guidelines for Good Manufacturing Practices (GGMP) [13]. Within FC, there are an inherent number of variation sources as shown in Figure 1, from upstream sample preparation through to data analysis, as well as the choice of statistics used. Hence operator perception and decision making can increase the variability of data being gated and analysed at a simplistic, univariate level. When faced with analysing highly dimensional, compounding data, the variability in output may potentially correlate with the complexity and difficulty of the data in question. However, ISO 15189 and the ICH GGMP currently do not recognise or accommodate for inter operator variability.

\subsection{Software processing}

Software exists to assist with the subjectivity of gate application by human operators [14], with many automated analysis platforms accessible for use applying initial gates based on a variety of statistical clustering and Principal Components Analysis $(P C A)^{5}$ methods. Gating-ML (extensible Markup Langauge) facilitates inter-site comparison of data by creating a file type that transfers pre-applied gates to data [15]. Various platforms and open source code software such as SPADE (1,2 or 3), RMeans and FlowClust, are

\footnotetext{
${ }^{4} \mathrm{ICH}=$ International Committee for Harmonization

${ }^{5}$ PCA $=$ Principal Components Analysis
} 
available to group FC data to identify subsets based upon location of raw data values [16].

Whilst these partially replace the operator when identifying subsets, they can be difficult to implement when the data is not easy to separate, often requiring manual checks postprocessing. There will also potentially be variability in the end results produced from different analysis software, due to differences in algorithms, coding methods and statistical analysis applied.

\subsection{Analysis standards}

The International Society of Analytical Cytology (ISAC) $)^{6}$ champions standards to aid gating in the Flow Cytometry field; known as Optimised Multicolour Immunofluorescence Panels $(\mathrm{OMIPS})^{7}$ [17]. These Panels contain significant guideline information relating to the setup, staining and analysis of the cells for immunophenotyping and characterisation of specific immunological markers within certain disease states. As such they are a useful model guideline series, even if the content may not be specific to the analysis being conducted. However, they do contain information relating to antibody clone and suggested fluorophore combinations for targets so do contribute to standardisation through both documentary and physical means. However, even following the gating protocols in these standards, the authors have observed that there still remain elements of subjective interpretation.

\subsection{External Quality Assessment}

Various clinical External Quality Assessment Schemes (EQAS) ${ }^{8}$ exist globally to monitor variability and performance of multiple clinical and pathology centres analysing blood samples using Flow Cytometry [18]. UK NEQAS runs multiple schemes for different enumeration and classification assays and defines performance boundaries based upon

\footnotetext{
${ }^{6}$ ISAC = International Society of Analytical Cytology

${ }^{7}$ OMIPS = Optimised Multicolour Immunofluorescence Panels

${ }^{8}$ EQAS = External Quality Assessment Schemes
} 
187 the observed distribution and variation of data across centres when analysing standard

188 samples. Historically, $95 \%$ Confidence Intervals or $10 \%$ trimmed mean \pm 2 Standard

189 Deviations (SD) ${ }^{9}$ have been used to specify data falling outside control limits, and so 190 identify centres for retraining. This facilitates continuous improvement and lowers overall 191 variation over time [18,19], but importantly EQA schemes do not identify sources of 192 uncertainty or evaluate uncertainty budgets. Through discussion with UK NEQAS, it has 193 been further reinforced that subjective interpretation is prevalent within FC manual gating. 194

However, when applying similar control limits or error estimators to patient data or a cell therapy product, 'outliers' will be common within the probability distribution of the data (parametric or non-parametric) and cannot be excluded because they represent a specific patient or patient data upon which decisions must be based. Previous work has shown that patient data for cell counts for cell therapy starting materials can vary by up to 4 orders of magnitude with positively skewed distributions, which potentially creates significant difficulty when manufacturing a product that has to cope with this incoming variation [20].

\subsection{Control limits for manufacture}

205 When defining control limits for product manufacture and release, validation is usually 206 based upon historical data sets and company specifications. Differences in these bounds 207 rely on correct analysis of population spread, to infer the correct location and spread 208 parameters. Methods can be broken down into three statistical approaches; Normal 209 statistics outlier estimators, Robust statistics outlier estimators, Multivariate statistics 210 outlier estimators. 
212 Normal distribution statistics are most commonly used to describe data, but this causes

213 problems when distributions are heavily skewed and non-parametric in shape. Log

214 transformation of this data is a traditionally common method employed to negate analysis

215 issues with differing data spreads, but when critical Quality Control Indicators (QCI)

216 decisions rely on data output, as well as the outcome of patient data regarding treatment,

217 it is best to focus on the raw data to ensure that resolution and discrimination are accurate. 


\subsection{Statistical Analysis Methods}

220 Determining outliers is completed by calculation of location and spread values of a data 221 set in question. Location parameters such as the mean, median or trimmed means are 222 points used to represent a population and spread parameters such as Standard Deviation 223 or Absolute Deviation represent the amount of fluctuation around the defined location 224 parameter. Historically, boundaries such as Mean \pm 2 SD have been used, because they approximately align to a 95\% Confidence Interval, assuming normally distributed data.

\subsection{Normal statistics outlier estimators}

These parameters can be calculated with any substantial data set, as long as their distribution represents that of the raw data in question. Often, normal distribution statistics are used to process and represent data that may be non-parametric in nature, potentially leading to misinterpreted information and a skewed judgement, conclusion or pass/fail decisions. Robust statistics are an alternative, opting to use medians instead of means, for example when data has a fat tail and mean values are often positioned further along the tail than the highest frequency point. Using median location values negates this problem.

Table 1 identifies various outline boundaries that are based upon normal distribution and robust statistics, found to be used across many examples of FC analysis and within other industries. Whilst this is not an exhaustive list, the majority use univariate data to understand distributions. Regression analysis requires two input variables to determine a $95 \%$ Confidence Interval, whereas Mean \pm 3SD are traditional bounds, originally championed in the use of control charts to monitor process quality over time [21]. These have been amended for use in analytical chemistry QC [22] but were refined to Mean \pm 2SD in the 1990's FC EQAS [6,7,23], alongside use of 95\% Confidence Intervals, defined by Standard Error of the data set. 


\begin{tabular}{|c|c|c|c|}
\hline Error Boundary Estimator & $\begin{array}{l}\text { Normal/Robust } \\
\text { Statistical Methods }\end{array}$ & $\begin{array}{l}\text { Data } \\
\text { Dimensionality }\end{array}$ & $\begin{array}{l}\text { Outlier Limits/ } \\
\text { Accommodates Data }\end{array}$ \\
\hline $\begin{array}{l}\text { Shewhart's Control Charts } \\
\text { Mean } \pm 3 S D\end{array}$ & Normal & 1 parameter & Outlier limits \\
\hline EWMA charts & Robust & 1 parameter & Outlier limits \\
\hline Mean $\pm 2 S D$ & Normal & 1 parameter & Outlier limits \\
\hline Calculation from Type I errors & Normal & 1 parameter & Outlier limits \\
\hline $10 \%$ trimmed Mean $\pm 2 S D$ & Normal & 1 parameter & Outlier limits \\
\hline Median $\pm 2 M A D$ & Robust & 1 parameter & Outlier limits \\
\hline M-Estimators & Robust & 1 parameter & Accommodates data \\
\hline Paxton's Criterion & Robust & 1 parameter & Outlier limits \\
\hline Linear regression models & Normal & $\begin{array}{l}\text { Minimum } 2 \\
\text { parameters }\end{array}$ & Outlier limits \\
\hline $\begin{array}{l}\text { Robust Multivariate } \\
\text { regression }\end{array}$ & $\begin{array}{l}\text { Robust, but transforms } \\
\text { data to normal }\end{array}$ & $\begin{array}{l}\text { Minimum } 2 \\
\text { parameters }\end{array}$ & Outlier limits \\
\hline $\begin{array}{l}\text { Longitudinal mixed effects } \\
\text { models }\end{array}$ & Robust & $\begin{array}{l}\text { Minimum } 2 \\
\text { parameters }\end{array}$ & Outlier limits \\
\hline Bootstrapping & $\begin{array}{l}\text { Normal, or log } \\
\text { transformation of non- } \\
\text { normal data }\end{array}$ & 1 parameter & Outlier limits \\
\hline $\begin{array}{l}\text { Euroflow } \\
\text { Personalised outlier estimator }\end{array}$ & $\begin{array}{l}\text { Robust, but transforms } \\
\text { data to normal }\end{array}$ & 1 parameter & Outlier limits \\
\hline
\end{tabular}

Table 1: Common outlier estimator methods used within Flow Cytometry and other industries. 


\subsection{Robust statistics outlier estimators}

252 When considering robust statistics, the Exponentially-Weighted Moving Average

$253(E W M A)^{10}$ Chart (Table 1$)$ is an alternative to the Mean $\pm 3 S D$ method, that adjusts to the 254 data set and is often preferred when detecting small shifts in components [24]. The use of 255 EWMA charts to track data has been recommended more as an internal assessment [25], 256 because it can be easily understood in a similar manner to a Levy-Jennings plot, and for 257 this reason has not been used for analysis or plotted in subsequent figures. Trimmed 258 means are another alternative location parameter, involving recalculation of new spread 259 metrics from a refined data set removing a certain percentile of data from the extremes, 260 making the distribution more centralised [25]. This has been popular in the UK NEQAS FC 261 Schemes [26,27], allowing pathology laboratories identified as extremes to improve over 262 time. However, if used incorrectly it can remove extremes that require investigation into 263 causality, and still represent probable events occurring.

A robust alternative to the Mean $\pm 2 S D$, is the Median \pm 2 Median Absolute Deviations $(M A D)^{11}[28]$. This uses the Median value of residuals from the location median to estimate spread, which is more aligned to non-parametric distributions. An additional traditional 268 robust method was described for an early FC EQAS (Paxton's Criterion), that uses 269 parameters calculated to also define Box and Whisker plots, from $25^{\text {th }}$ and $75^{\text {th }}$ percentiles, 270 and subtraction and addition of the Interquartile Range respectively [29]. Bootstrapping is 271 a resampling method used as an alternative technique for validation $[30,31]$ that derives confidence intervals, making assumptions on the data probability distribution, and

273 assumes a normal distribution or uses a log-transform of non-normal data. This is a 274 suitable way of defining bounds if it fits the raw data or if the transform sufficiently equates.

\footnotetext{
${ }^{10}$ EWMA = Exponentially-Weighted Moving Average

${ }^{11}$ MAD = Median Absolute Deviation
} 


\subsection{Multivariate statistics outlier estimators}

277 As previously mentioned, regression analysis is a common method for specifying data278 fitness bounds. Linear regression analysis (Table 1) minimises the residual sum of 279 squares and uses residual plots to detect outliers. Linear regression has been used to 280 compare FC EQAS approaches [32], with further developments building upon this by using 281 robust multivariate regression [33], which is less sensitive to outliers due to transformation 282 of non-normal data.

284 Longitudinal mixed effects models have been employed more recently by UK NEQAS $285[34,35]$. This uses a mixture of pass criteria based upon different residual and deviate 286 values, allowing for multiple stratification levels of the data, based upon robust statistics. 287 An alternative method uses M-estimators, that accounts for all of the data, but weights 288 extreme values to shift them to an optimal boundary, also known as Winsorizing means. 289 This does not technically exclude any data points as previously described methods do, but 290 still uses normal statistics to define initial boundary points. 


\subsection{Histogram File Generation for this Study}

293 A series of .fcs 3.0 files were generated using a BD Bioscience FACSCanto II Flow 294 Cytometer with 4-2-2 optical configuration, by running a suspension of BD Bioscience Cytometer Setup and Tracking $(C S \& T)^{12}$ beads [36]. The CS\&T beads were a mix of dim, medium and brightly fluorescing polystyrene beads and are used to monitor baseline and daily performance of FC instruments. The beads were selected for use due to their lesser variation compared to biological variance. The beads were run through the allophycocyanin $(\mathrm{APC})^{13}$ channel, excited by the red laser $(633 \mathrm{~nm})$. The APC channel was used, because it is a common channel across a wide variety of FC instruments.

Voltages, and therefore fluorescence peaks, are adjusted to determine the optimal analysis parameters in line with instrument sensitivity. The gain can be changed by voltage setting to alter the position of the fluorescence peaks seen through the APC channel on a univariate scale. Many methods have been developed to determine Flow Cytometry analysis parameters, but the most popular, referred to as the 'Peak 2 method' involves running a control sample at different voltage intervals and calculating the stain index [37].

The .fcs files generated for this work (Figure 2) were gathered at ten different voltages from $250 \mathrm{~V}$ to $700 \mathrm{~V}$, in $50 \mathrm{~V}$ intervals, allowing for resolution differences to be seen whilst not over-exerting the concentration of operators involved in the study. The .fcs figure elements in Figure 2 show the progression of the three data peaks across the univariate scale, in relation to the gain increase of the APC channel of the Flow Cytometer. This figure also shows the range gates (red circle) applied to the histograms, which participants were asked to copy.

\footnotetext{
${ }^{12}$ CS\&T = Cytometer Setup \& Tracking

${ }^{13} \mathrm{APC}=$ Allophycocyanin
} 

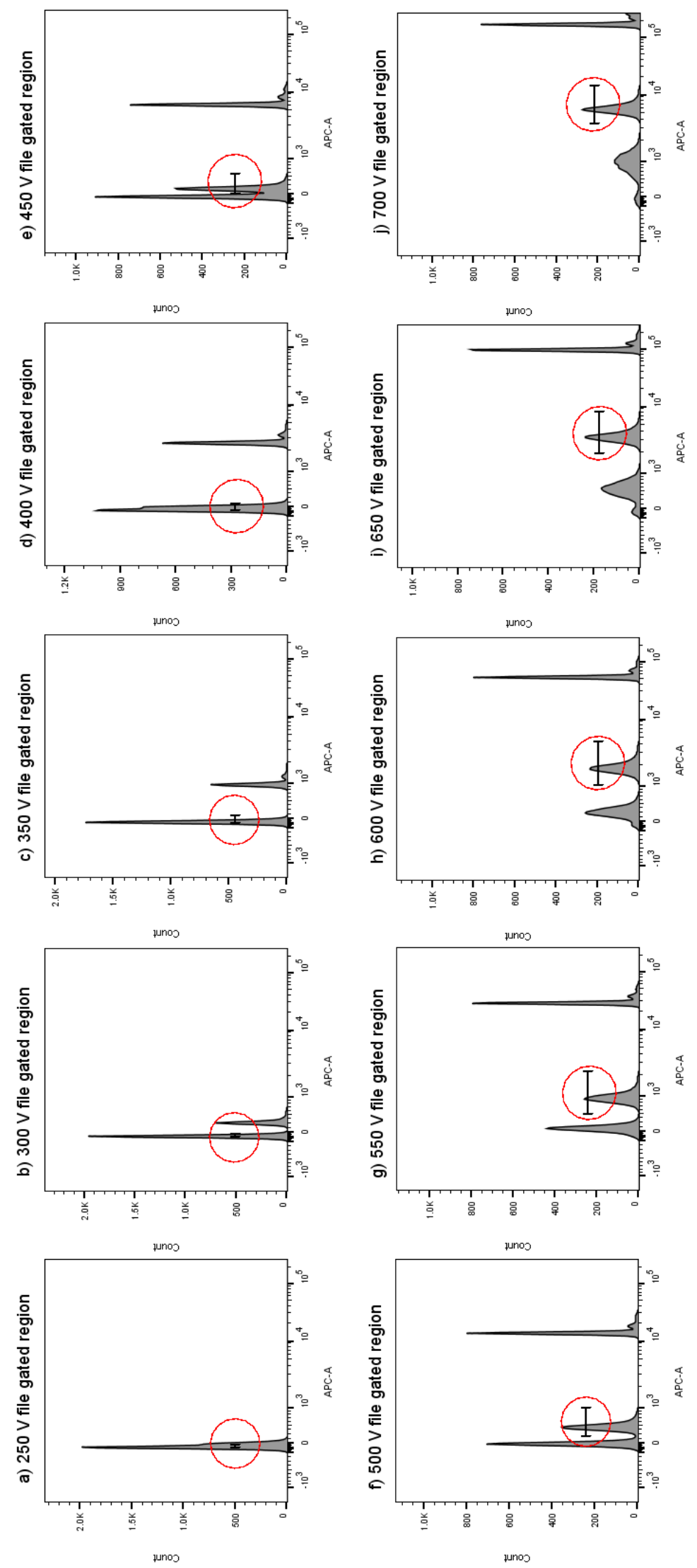

Figure 2 Images of histograms provided for participant gating studies ( $250 \mathrm{~V}$ to $700 \mathrm{~V}$ ) 


\subsection{Operator Study Methodology}

32136 participants were enrolled in this study to analyse the histogram files; 10 from an academic institution, 19 from an industrial cell therapy process development team and 7 as part of a data gathering exercise at the 2017 FIRM Symposium [38]. Ethical and GDPR approval and was obtained for this study by local University Human Participant Ethics SubCommittee. Participants completed the study individually and were asked to complete two phases of analysis. The two phases aimed to monitor the effects of personal data interpretation, in comparison to following a pre-defined protocol, or interpretation thereof, with the hypothesis of reduced inter-operator variation for FC analysis when following the 329 latter.

\subsection{Phase 1 Histogram gating - Operator judgement}

332 During Phase 1, participants were asked to apply range gates to the area of the plots where they estimated the medium fluorescing peak lay. Five of the plots were considered to have poor peak separation (Figure 2 a-e), where there was difficulty in discerning peaks. The remaining five files were considered to have good peak separation (Figure $2 \mathrm{f}-$

j) and to be more representative of good Flow Cytometry data. Participants used the thirdparty software FlowJo Version 10.0.8r1 to examine the .fcs files and apply histogram gates, using default bi-exponential scaling and maintaining the histogram view setting.

\subsection{Phase 2 Histogram gating - Protocol driven}

341 For Phase 2, participants were provided with printed images of range gates (highlighted by 342 the red circles in Figure 2) that had been pre-applied to the series of files and were asked 343 to adjust their gates to try and match these 'diagrammatical protocols'. These gates were 344 applied to aid precision of application, not accuracy, because accuracy can be translated 345 into different subject contexts. The poor gated files were included to demonstrate the 
346 potential impact and worth of using diagrammatical protocols in difficult analysis

347 conditions.

\subsection{Analysis metrics}

350 Data was statistically and graphically processed in IBM SPSS for Statistics Version 24 and 351 Matlab R2018. Range gates applied to histograms of one fluorescence channel show 352 variation in spread and location parameters, chosen by perception of the operator. The 353 Robust Coefficient of Variation $(r C V)^{14}$ (as defined in Eq. 1) is a commonly used metric 354 within FC data analysis to compare variation amounts between operators and 355 measurement phases. It helps the operator to understand and monitor variation within a 356 system, combining both location and spread parameters into one measure.

$$
r C V(\%)=\frac{r S D}{\text { Median }} \times 100
$$

These $r C V$ measures have been used to calculate a total range of variation between operators (inter-operator), per histogram file, per phase. Range was used to monitor reduction and therefore improvement in inter-operator variation attributed to the results, because it is an easily understood measure of spread and monitors the total range rather than an arbitrary measure of spread (such as standard deviation). The difference in range between operators can be used to observe the effectiveness of protocol instigation, with percentage of variation reduction monitored alongside this. Normality tests were completed in IBM SPSS using the Shapiro-Wilk test along with the generation of Standard Deviation (SD) data.

\footnotetext{
${ }^{14} \mathrm{rCV}=$ robust coefficient of variation
} 


\subsection{Analysis of Results}

369 The rCV range between operators measures as defined in Table 2, is higher in Phase 1 370 Operator judgment (Figure 3 - left image - blue line), compared to the lower inter-operator 371 range in Phase 2 - Protocol driven (Figure 3 - left image - orange line), apart from the 250

372 V file. This shows better separation between the stages at all file values, and the different

373 inter-operator ranges for all files. The inter-operator ranges were calculated as the

374 difference between the minimum and maximum $r C V$ values of all the operators gating

375 each peak within a certain file. The percentage reduction in range between operator

376 variation from Phase 1 to Phase 2 is shown by the bar chart in Figure 3 (right).

[Insert Figure 3 Here]

379
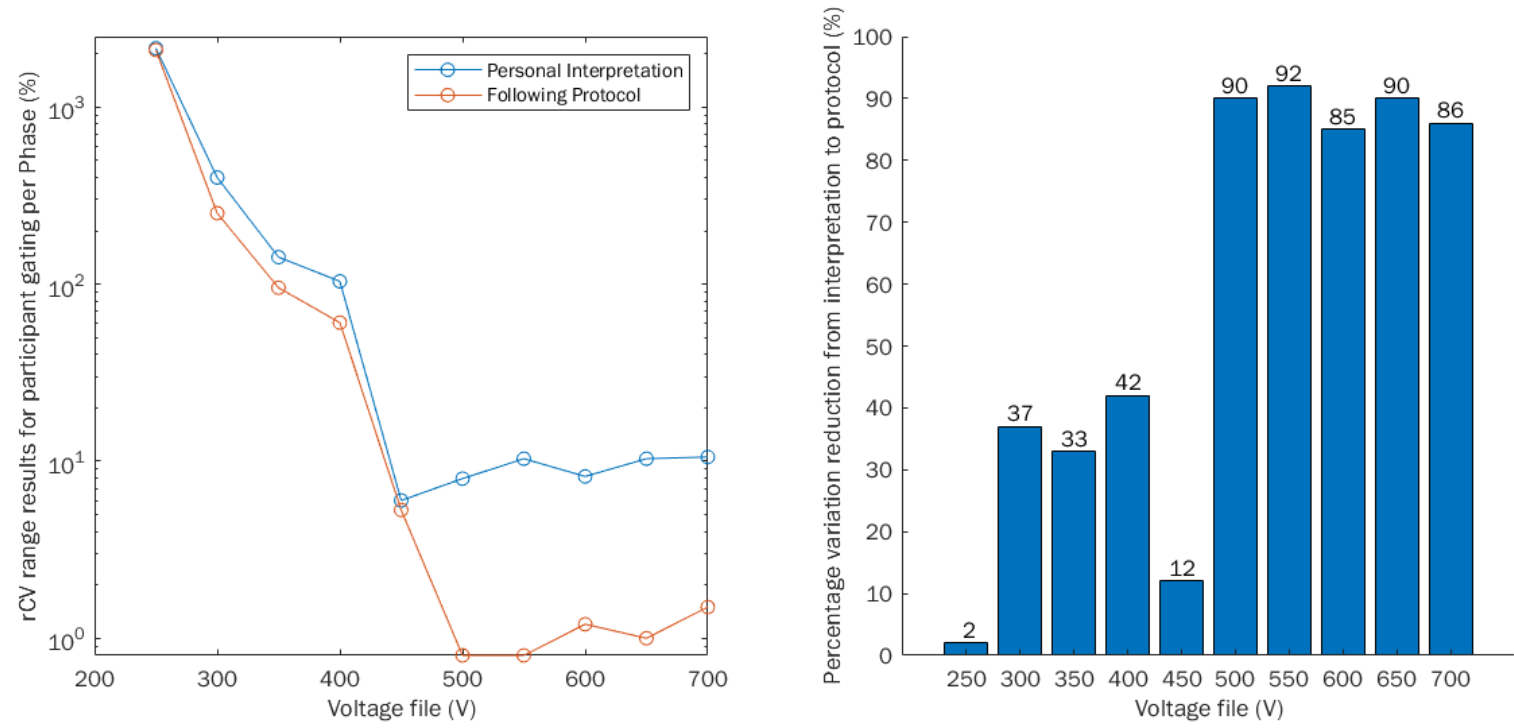

Figure 3: Comparison of inter-operator ranges of personal interpretation of histogram data

382 (left) and percentage reduction from Stage 1 to Stage 2 results (right) 


\begin{tabular}{|l|c|c|c|c|c|c|c|c|c|c|}
\hline Voltage (V) & 250 & 300 & 350 & 400 & 450 & 500 & 550 & 600 & 650 & 700 \\
\hline Figure 2 & $\mathrm{a}$ & $\mathrm{b}$ & $\mathrm{c}$ & $\mathrm{d}$ & $\mathrm{e}$ & $\mathrm{f}$ & $\mathrm{g}$ & $\mathrm{h}$ & $\mathrm{i}$ & $\mathrm{j}$ \\
\hline $\begin{array}{l}\text { Personal interpretation } \\
\text { inter-operator range } \\
\text { (FI) }\end{array}$ & 2150 & 400.0 & 142.0 & 104 & 6 & 7.6 & 10.3 & 8.2 & 10.3 & 10.6 \\
\hline $\begin{array}{l}\text { Phase 1 } \\
\text { Gating standard inter- } \\
\text { operator range (FI) }\end{array}$ & 2100 & 251.5 & 95.4 & 60.6 & 5.3 & 0.8 & 0.8 & 1.2 & 1.0 & 1.5 \\
\hline $\begin{array}{l}\text { Reduction (\%) } \\
\text { Phase 2 }\end{array}$ & 2 & 37 & 33 & 42 & 12 & 90 & 92 & 85 & 90 & 86 \\
\hline
\end{tabular}

Table 2: Inter-operator ranges

1.5 column width

391 It can be seen that the notionally 'good separation' files (Figure 3, $500 \mathrm{~V}$ to $700 \mathrm{~V}$ ) have a much greater reduction in variation between operators when they follow the diagrammatical protocol, reducing the amount of contributory variation to the end result.

395 In addition, when speaking to operators as they completed these studies, it became 396 apparent that less informed or experienced users sometimes applied gating knowledge 397 from other measurement techniques that generate peak spectra as measurement output 398 (e.g. Mass, Raman and Infrared Spectrometry). This practice may or may not have been 399 beneficial. 


\subsection{Optimum voltage for this Flow Cytometry data}

404 The optimum voltage for this set of data would be $500 \mathrm{~V}$. This method has been

405 demonstrated in Figure 4 [37], where the median operator $r C V$ values from each voltage 406 file in Phase 1 were plotted to create a Stain Index $r C V$ curve. Median values were plotted 407 for each file due to non-parametric distributions of operator results for files across Phase 1 408 and Phase 2 analysis. The inflection point that changes the curve to a plateau indicates 409 the optimum voltage, minimising the effect of background noise on the data. This voltage 410 file was selected to further investigate the distribution of an example data set, and the 411 effects of different statistical outlier methods (Section 3) for determining outliers and 412 control limits.

[Insert Figure 4 Here]

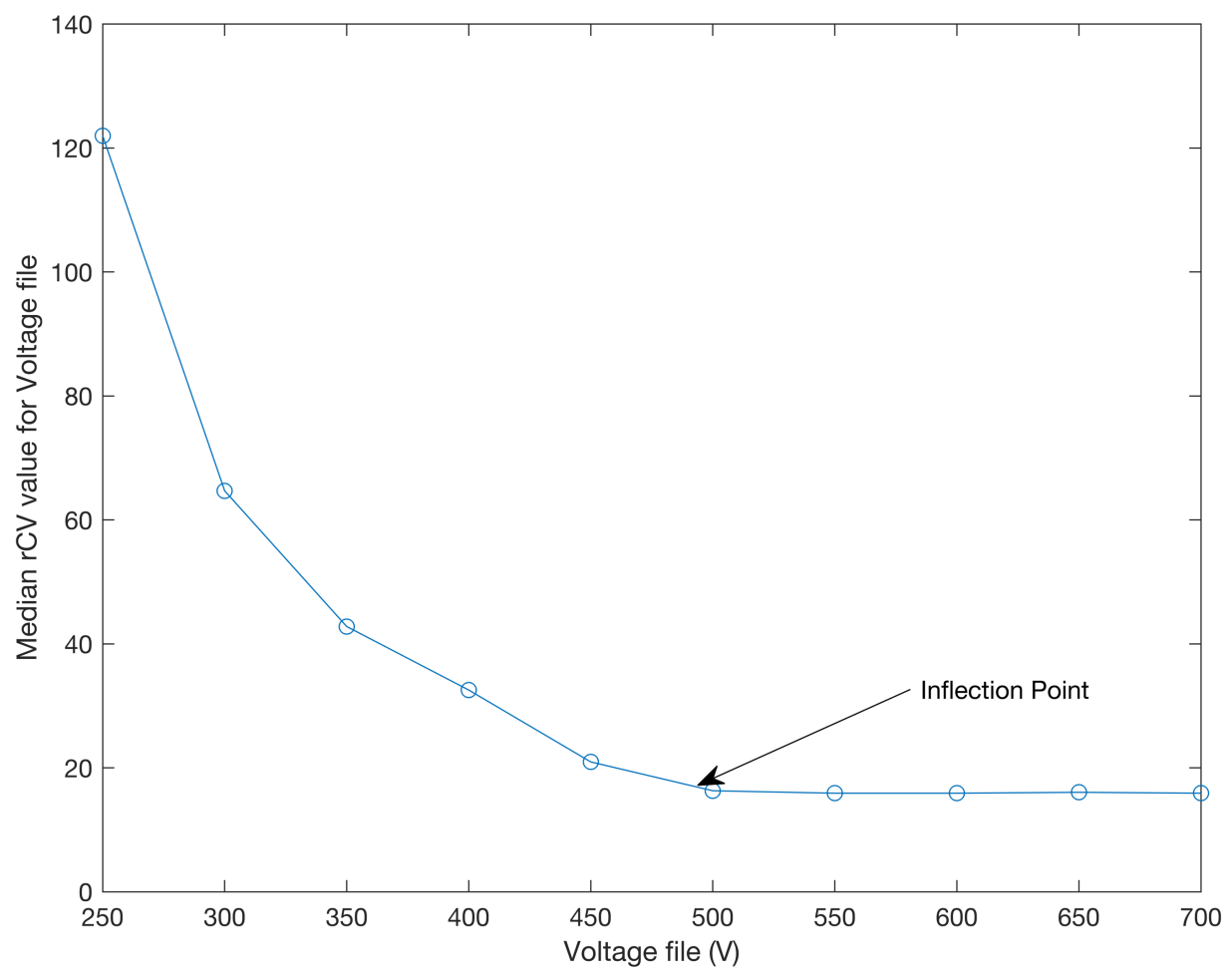

415 416

Figure 4: Stain Index $r C V$ curve.

1 column width. 
420 The variation for the $500 \mathrm{~V}$ file is reduced by $90 \%$ when operators used the

421 diagrammatical protocol. As the voltage files increase from $500 \mathrm{~V}$, and therefore the

422 separation between the peaks increases, the reduction in variation is still very high, but the

423 trend decreases slightly from this optimum analysis point. This could be due to the gain

424 increase amplifying background noise (as well as the fluorescence peaks), creating

425 additional shoulders on the peaks, which operators also gated on. This demonstrates the

426 importance of determining optimal settings to ensure that no other variation sources affect

427 interpretation of a final result and understanding where larger sources of uncertainty come 428 into play.

429

430 Lower voltages reduce sensitivity, which can be seen in the files by the merging peaks.

431 Consequently, it becomes harder to discern the peaks from each other, and from

432 background noise. The 'poor separation' files have reduced variation between operators

433 when they follow the diagrammatical protocol, but this is not as extensive as the clearly

434 separated files. This further supports the requirement for proper setup when analysing

435 data through a complex metrology instrument. 


\subsection{Understanding the impact of outlier discrimination}

439 Outlier discrimination has been explored using the $500 \mathrm{~V}$ Phase 1 analysis data as an 440 exemplar data set. Typically, within manufacturing analysis outliers or extreme values are

441 either removed from analysis or the outliers are addressed to facilitate future improvement 442 in process control. Table 1 summarizes different outlier estimator methods (boundary 443 specifications) used within a variety of industries including biomedical and cell therapy 444 biomanufacture. There is no one correct choice for outlier determination, with the choice 445 being based on commercial guidelines, quality standards, and/or method validation exercises.

To demonstrate the effect that different estimators have on data, the Phase $1500 \mathrm{~V}$ data set was used to calculate different outlier discrimination intervals. This data set was initially tested to describe the distribution, using IBM SPSS to calculate the descriptive statistics.

451 The Shapiro-Wilk test for departure of normality confirmed that the data set was nonparametric $(p<0.05)$, alongside a strong negative Skew $(-2.21)$ and high Kurtosis (5.27), making the results more affected by the presence of 'outliers'.

To apply different error boundaries to the data set, the outlier discrimination methods were screened to include those that could be applied to a univariate data set for data that is not manufactured-part specific, and those that act as a set boundary and do not transform the extreme values. Both normal distribution and robust statistics have been included. The

459 effect of logarithmic transformation on the data has also been investigated, because this is 460 a common method used to transform data to make better approximate to a normal 461 distribution. This allows non-parametric data to be more applicable to a wider range of 462 Gaussian-based statistical analyses and conforming to more traditional manufacturing statistical process control paradigms. 
465 The calculations used for both the raw and transformed data can be seen in Table 3.

466 Bootstrapping relies upon a 95\% Confidence Interval calculation, so these have been

467 accounted for together, and Mean $\pm 3 S D, 2 S D$, and $10 \%$ trimmed mean \pm 2 SD all use the 468 same SD calculation to generate control limits.

469

470

[Insert Table 3 Here] 


\begin{tabular}{|c|c|c|c|c|c|c|c|c|c|c|}
\hline $\begin{array}{l}\text { Outlier } \\
\text { estimator }\end{array}$ & $\begin{array}{l}\text { Location } \\
\text { Calculation }\end{array}$ & Spread calculation & $\begin{array}{l}\text { Location } \\
\text { value }\end{array}$ & $\begin{array}{l}\text { Spread } \\
\text { value }\end{array}$ & $\begin{array}{l}\text { Lower } \\
\text { Control } \\
\text { Limit }\end{array}$ & $\begin{array}{l}\text { Upper } \\
\text { Control } \\
\text { Limit }\end{array}$ & $\begin{array}{l}\text { Control } \\
\text { Limit } \\
\text { Distance }\end{array}$ & $\begin{array}{l}\text { Log } \\
\text { transformed } \\
\text { Lower } \\
\text { Control Limit }\end{array}$ & $\begin{array}{l}\text { Log } \\
\text { transformed } \\
\text { Upper } \\
\text { Control Limit }\end{array}$ & $\begin{array}{l}\text { Log } \\
\text { transformed } \\
\text { Control Limit } \\
\text { Distance }\end{array}$ \\
\hline $\begin{array}{l}\text { Shewhart } \\
\text { Control Charts } \\
\text { Mean +/- 3SD }\end{array}$ & \multirow{5}{*}{$\begin{array}{l}\text { For trimmed } \\
\text { data, } 10 \% \text { of } \\
\text { the ordered } \\
\text { data are } \\
\text { removed } \\
\text { before } \\
\text { calculation }\end{array}$} & \multirow{3}{*}{$s= \pm \sqrt{\frac{\sum\left(x_{i}-\bar{x}\right)^{2}}{n-1}}$} & \multirow[t]{2}{*}{15.59} & 5.16 & 10.43 & 20.75 & 10.32 & 1.02 & 1.36 & 0.34 \\
\hline Mean +/- 2SD & & & & 3.44 & 12.15 & 19.03 & 6.88 & 1.08 & 1.30 & 0.23 \\
\hline $\begin{array}{l}10 \% \text { trimmed } \\
\text { mean }+/-2 S D\end{array}$ & & & 15.88 & 3.44 & 12.44 & 19.32 & 6.88 & 1.09 & 1.32 & 0.23 \\
\hline $\begin{array}{l}95 \% \\
\text { Confidence } \\
\text { Intervals }\end{array}$ & & \multirow{2}{*}{$C I= \pm 1.96 \frac{s}{\sqrt{n}}$} & 15.59 & 0.55 & 15.04 & 16.14 & 1.10 & 1.17 & 1.21 & 0.04 \\
\hline Bootstrapping & & & & & & & & & & \\
\hline Med +/-2MAD & \multirow{2}{*}{$\begin{array}{l}\text { Median } \\
=\left(\frac{n+1}{2}\right)\end{array}$} & $M A D=\operatorname{med}\left(\left|x_{i}-\operatorname{med}(x)\right|\right)$ & 16.30 & 0.80 & 15.50 & 17.10 & 1.60 & 1.19 & 1.23 & 0.04 \\
\hline IQR Metrics & & $\begin{array}{c}I Q R=75 \text { th \%ile }-25 \text { th } \% \text { ile } \\
L C L=25 \text { th \%ile }-I Q R \\
U C L=75 \text { th \%ile }+I Q R\end{array}$ & & & 14.20 & 17.80 & 3.60 & 1.16 & 1.25 & 0.10 \\
\hline
\end{tabular}

\section{2 column width}


474 Figures 5 and 6 show plots representing these non-parametric and transformed results.

475 Figure 5 shows two histograms of operator $r C V$ values for the $500 \mathrm{~V}$ file, where operators

476 were asked to apply gates based upon personal interpretation (upper histogram), and then

477 asked to follow protocols (lower histogram). The grey stepped bands represent the

478 calculated outlier discrimination boundaries derived using the information in Table 3. Any

479 histogram column falling within these boundaries is determined as being in specification.

480 Those falling outside of a boundary would then be classified as an outlier by that method.

481 The majority of participants fall within all bounds, but this shows the importance of correct

482 selection of discrimination for the process. If chosen control limits are too small, then

483 allowable variation could be cut out, causing a high defect rate and false negatives. Too

484 large, and the process is allowed to head towards a more out-of-control state and false

485 positives could be included in analysis, which is undesirable and makes location of the 486 source of the variation more difficult. 

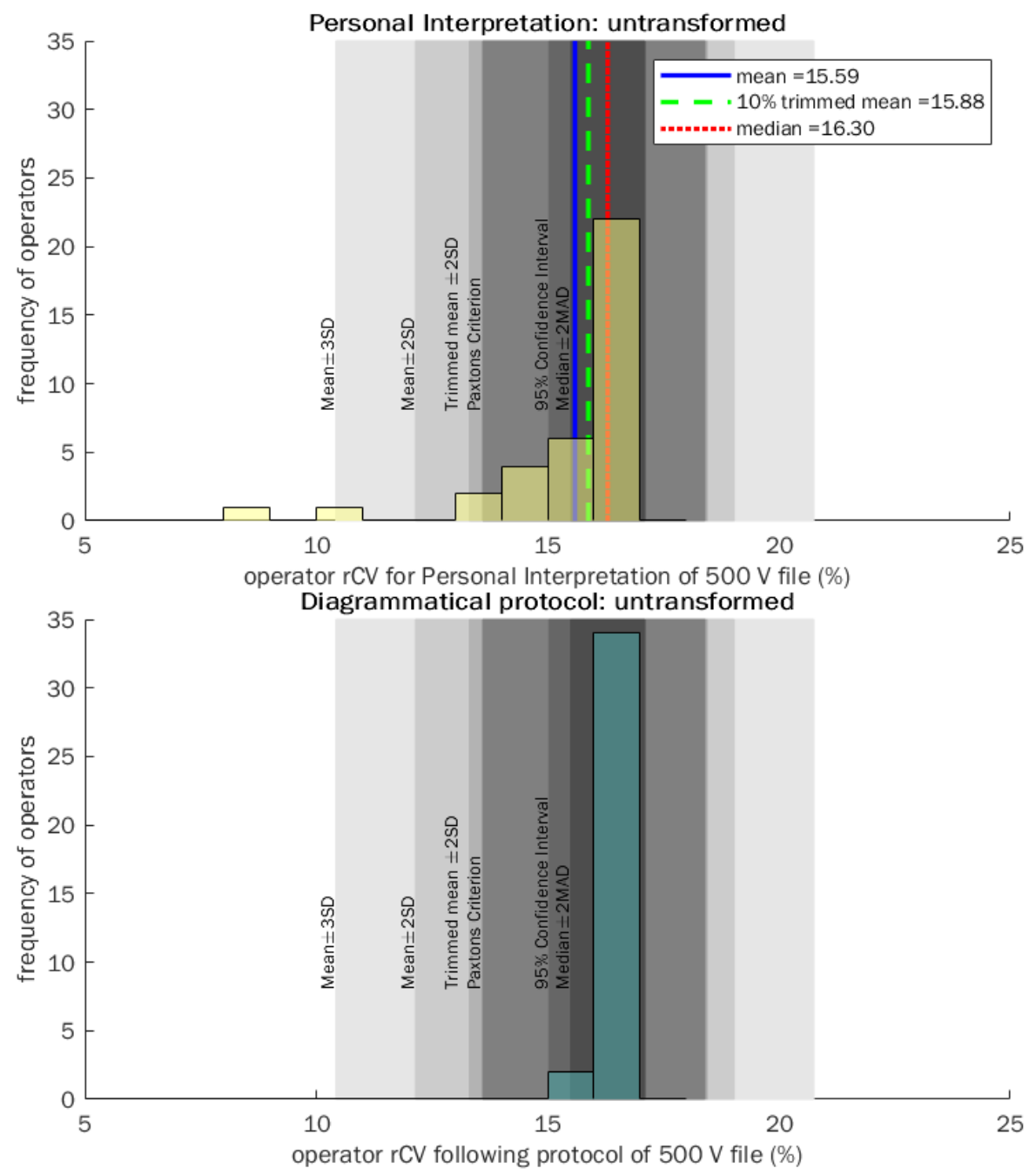

489 Figure 5: Application of different control limits applied to raw data

2 column width.

492 To show the impact of operators following use of a gating standard to obtain results, the

493 Phase 2 data was overlaid onto discriminatory bounds, as seen in Figure 5 (lower

494 histogram). All participants fall within the tightest boundary, specified by the Median \pm 4952 MAD. This confirms that causing operators to follow a visual protocol not only reduces 496 inter-operator variation but also reduces the chance of 'outlier' results, regardless of how 

outliers are defined in this context. In the context of cell therapies and their manufacture,

498 these outliers will represent key patient data metrics and cannot be excluded.

\section{[Insert Figure 6 here]}
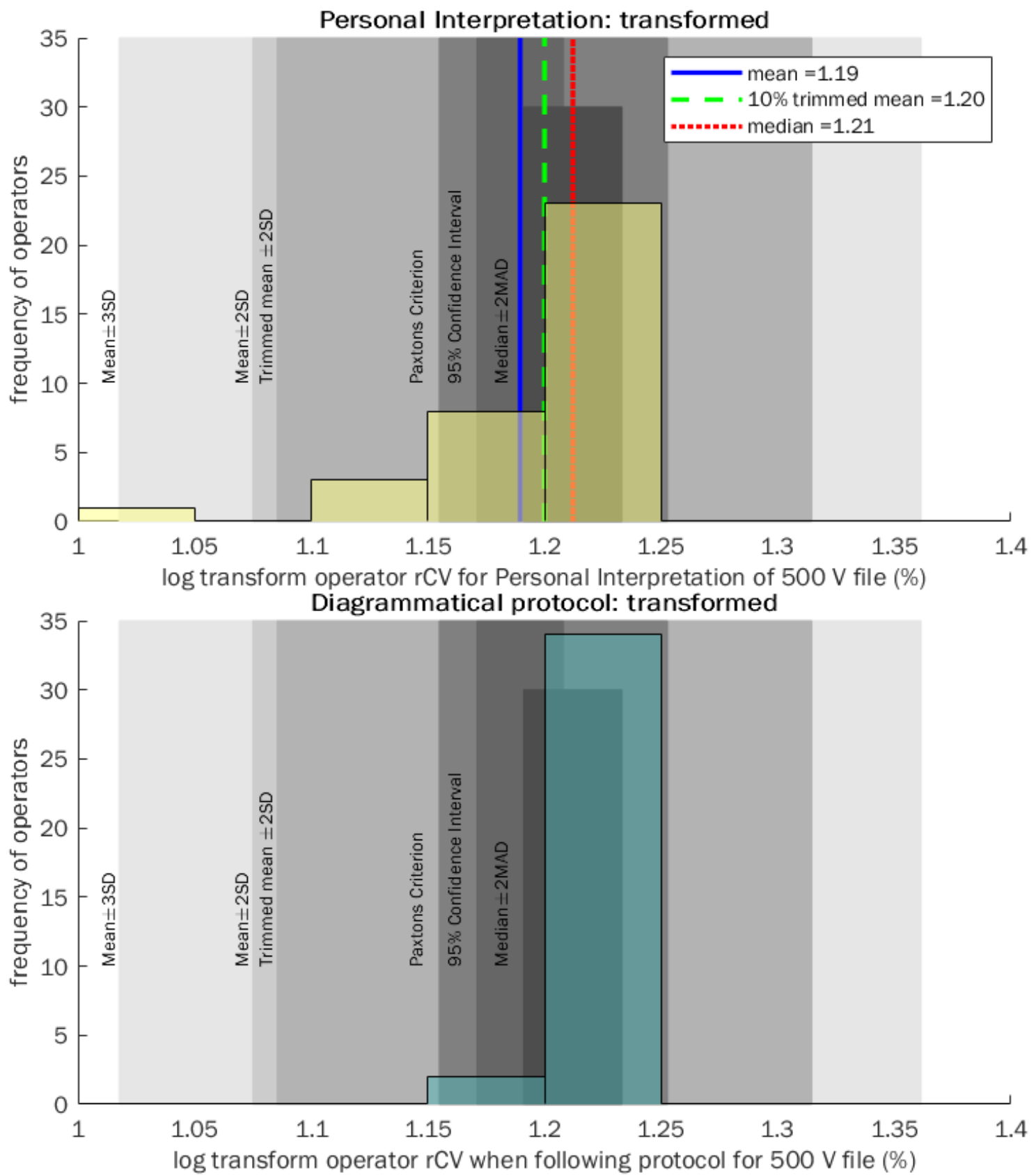

501 Figure 6: Application of different control limits applied to log transformed data 
As seen in Figure 5, all boundaries scale inwards and outwards, so overlaps can be seen on both sides. The mean, $10 \%$ trimmed mean and median have all been plotted on top, to show shift due to the distribution. The median value of the data fits best to represent the skewed distribution because it sits within the histogram maxima.

The boundaries in Figure 6 were recalculated when the skewed data was normalised with a log transform (noting the change of $x$-axis scaling). The outer boundaries in Figure 6 (upper histrogram) all fall within the same gradient pattern towards the centre, however the $95 \%$ confidence interval and the Median $\pm 2 M A D$ bounds sit astride one another, discounting various operators as outliers. Application of log transformed Phase 2 data to these defined boundaries is shown again in Figure 6 (lower histogram), with operators falling within the tightest boundaries when following a diagrammatical protocol to apply their gates.

\section{To monitor equality of outlier discrimination between the bounds specified for raw and} transformed data, Table 4 shows the different bounds used, and what outliers are specifically discriminated against using this method (outliers identified using anonymous coding). Log transformation is deemed appropriate in the context here where the outliers are identical for each context and therefore labelled as 'Equal'.

\section{All outlier boundaries calculated up to and including Paxton's Criterion are equal in the} number of outliers specified when using either the raw data or log transformed data. As these boundaries become more refined, outliers are included due to the area of the peak gated by the specific operators. Mean $\pm 3 S D$ has one outlier quantified by the gate applied covering a small section of the peak. This does not include all events collected within the peak and therefore has a skewed $r C V$ value as a result. Mean $\pm 2 S D, 10 \%$ Trimmed Mean 
$530 \pm 2$ SD and Paxton's Criterion have all specified outliers due to gates not covering the

531 entirety of the peak, and the gradual inclusion of these outliers through more refined

532 control limits sees these outlier gates become wider to include more of the peak,

533 questioning the outliers have been defined due to differences in spread definition.

[Insert Table 4 Here]

\begin{tabular}{|c|c|c|c|c|c|c|}
\hline & $\begin{array}{l}\text { Outlier } \\
\text { frequency }\end{array}$ & Outlier code & $\begin{array}{l}\text { Log transformed } \\
\text { outlier } \\
\text { frequency }\end{array}$ & Outlier Code & $\begin{array}{l}\text { Equal } \\
\text { Outliers }\end{array}$ & $\begin{array}{l}\text { Reason for } \\
\text { outlier }\end{array}$ \\
\hline Mean $\pm 3 S D$ & 1 & $\mathrm{C} 4$ & 1 & $\mathrm{C} 4$ & EQUAL & $\begin{array}{l}\text { Range gate } \\
\text { applied over } \\
\text { smaller \% of } \\
\text { peak }\end{array}$ \\
\hline Mean $\pm 2 S D$ & 2 & $\mathrm{C} 1, \mathrm{C} 4$ & 2 & $\mathrm{C} 1, \mathrm{C} 4$ & EQUAL & $\begin{array}{l}\text { Range gate } \\
\text { applied over } \\
\text { smaller \% of } \\
\text { peak }\end{array}$ \\
\hline $\begin{array}{l}10 \% \text { trimmed } \\
\text { mean } \pm 2 S D\end{array}$ & 2 & $\mathrm{C} 1, \mathrm{C} 4$ & 2 & $\mathrm{C} 1, \mathrm{C} 4$ & EQUAL & $\begin{array}{l}\text { Range gate } \\
\text { applied over } \\
\text { smaller \% of } \\
\text { peak }\end{array}$ \\
\hline $\begin{array}{l}\text { Paxton's } \\
\text { Criterion }\end{array}$ & 5 & $\begin{array}{l}\text { A3, B11, C1, C4, } \\
\text { C7 }\end{array}$ & 5 & $\begin{array}{l}A 3, B 11, C 1, C 4, \\
C 7\end{array}$ & EQUAL & $\begin{array}{l}\text { Range gate } \\
\text { applied over } \\
\text { smaller \% of } \\
\text { peak }\end{array}$ \\
\hline $\begin{array}{l}95 \% \\
\text { Confidence } \\
\text { Interval }\end{array}$ & 29 & $\begin{array}{l}\text { A1-A10, B1-B5, } \\
\text { B7,B8, B10-B13, } \\
\text { B16, B17, C1, } \\
\text { C3-C7 }\end{array}$ & 28 & $\begin{array}{l}\text { A1- A5, A7-A10, } \\
\text { B1-B5, B7, B8, } \\
\text { B10-B13, B16, } \\
\text { B17, C1, C3,-C7 }\end{array}$ & UNEQUAL & $\begin{array}{l}\text { Additional outlier } \\
\text { in raw data (A6) }\end{array}$ \\
\hline $\begin{array}{l}\text { Median } \pm \\
2 \text { MAD }\end{array}$ & 9 & $\begin{array}{l}\text { A3, A5, A6, B4, } \\
\text { B11, B15, C1, } \\
\text { C4, C7 }\end{array}$ & 11 & $\begin{array}{l}\text { A3, A5, A6, B4, } \\
\text { B9, B11, B14, } \\
\text { B15, C1, C4, C7 }\end{array}$ & UNEQUAL & $\begin{array}{l}\text { Additional } \\
\text { outliers in log } \\
\text { transform (B9, } \\
\text { B14) }\end{array}$ \\
\hline
\end{tabular}


5372 column width

538

539 Both highly refined control limits (95\% Confidence Interval and Median $\pm 2 M A D)$ have an 540 unequal number of outliers when boundaries are calculated with raw and log transformed

541 data. When calculating the $95 \%$ Confidence Interval the raw data contained one extra 542 outlier, which may be expected due to this being a statistical test using normal metrics 543 applied to a non-parametric data set. Both the raw and log-transformed control limits for 544 the $95 \%$ Confidence Interval contain a high number of outliers (29 and 28 respectively), 545 leaving only 8 and 9 respective 'inliers' to represent the distribution. Due to the distribution 546 having a high Kurtosis and Skew, this could affect the calculation of this confidence 547 interval, due to its dependence on the standard deviation that is not located at the peak 548 maxima.

550 The robust Median \pm 2 MAD control limits are unequal due to two extra outliers being 551 present in the log transformed data. One could assume that this is because a robust 552 method has been applied to 'normalized' data. However, this should still be effective in 553 analysis despite of distribution shape. 


\subsection{Conclusion}

556 Flow Cytometry is a core biometrology technique within clinical, research and the newly 557 defining Cell Therapy biomanufacturing industry. Subjective manual interpretation of FC 558 data is commonly practiced, however this subjective manual interpretation is a key 559 potential source of process control input variation and hence measurement uncertainty in 560 Cell Therapy manufacture. Reducing variability within Cell Therapy manufacture will help 561 to optimise the process and to better understand with greater certainty where variation 562 comes from, if the process fluctuates and exceeds control limits.

When considering manual FC data analysis, this univariate gating study results shows for the first time that significant inter-operator reduction occurs when operators follow a diagrammatical protocol, with up to $90 \%$ decreases in $r C V$ values if operators are presented with clearly separated peaks. If peak separation is reduced then benefit gain 568 from diagrammatical protocols is likewise reduced due to poorer peak discrimination by the operators, even given a guiding protocol. Sensitivity thresholds require correct definition, 570 but over-amplifying to try and aid data definition can cause amplification and spill-over of 571 noise into identified measured regions.

573 As is the case in other industrial sectors, this supports the implementation and use of 574 standardised gating protocols in the field such as OMIPS for inter-site comparison. This 575 demonstrates one manner in which clinical but most importantly biomanufacturing centres, 576 can reduce biometrology variability within a Cell Therapy manufacturing context from data 577 analysis within Quality Control, although context for scale-up would require investigation.

579 Furthermore, this work has considered the importance of correct definition of control and/or specification limits, in the context of the assumed shape of data distribution within a 
581 specific process, either normalised or non-parametric. Formal method validation

582 traditionally defines these control limits, but in Cell Therapy manufacturing this can be 583 difficult on a patient-by-patient basis, due to lack of material available to complete multiple 584 replicates, along with the key point that notional 'outliers' cannot be discarded due to this 585 being true patient data.

587 The analysis of the $500 \mathrm{~V}$ exemplar data set has shown how selection of different control 588 limits (normalised and non-parametric based) leads to different definitions of outliers, with 589 some outliers being common to several control limits, whilst other outliers being unique to 590 a particular method. Given that there is minimal consensus as to selection and application 591 of the most appropriate control limit, this again exemplifies the potential for further variation 592 input within the biometrology feedback into a manufacturing process control scenario. In 593 addition, the patient specific nature of outliers in cell therapy statistical datasets is further 594 hindered by control limit selection and application.

Previous data from both healthy and diseased patients would be used to define Cell 597 Therapy manufacturing control limits, but there cannot be complete confidence as to 598 whether new patient data meets historical data sets and disease states are often much 599 more variable. This makes definition of initial control limits difficult. Recent EU guidelines 600 state a minimum of 3 validation runs are required to define control limits before a therapy 601 receives Market Authorization Approval (MAA) ${ }^{15}$ [39], with manufacturers taking a Risk602 based approach to validation. This number of validation runs is lower than other more 603 traditional industrial validation scenarios, but MAA would ensure manufacturers are aware 604 of additional variation sources in the Cell Therapy manufacturing process and the 605 uncertainty quantification of these. The better the control of variation of the biometrology

\footnotetext{
${ }^{15}$ MAA = Market Authorization Approval
} 
technique, the more assurance that any observed variation more probably originates from

607 the biological material and biomanufacturing process. This allows decisions to be made 608 with more confidence within Quality Control Processes.

609

This study has provided initial awareness of operator behaviour and the potential for

611 variation within subjective manual Flow Cytometry data processing. This will directly inform

612 the definition of operator analysis studies using simple to highly complex cell models and

613 datasets (in terms of significantly increasing dimensionality), with different gating scenarios

614 designed to quantify the variation present when gating clearly separated and poorly

615 separated cellular populations. However, it is important to recognise that software-based

616 auto-gating techniques may also be used for Flow Cytometry data analysis. Whilst not fully

617 automated in the true sense of the word, auto-gating techniques may aid the operator

618 during the data analysis. As yet, auto-gating techniques have not been assessed in a

619 comparable manner to these studies of operator manual data analysis.

621 In addition, the work reported here is not intended to develop or deliver a whole FC

622 instrument uncertainty statement. It is fully anticipated that the results of the operator

623 variation analysis will eventually inform a detailed operator uncertainty analysis, and

624 subsequently contribute to a whole FC instrument uncertainty statement. 


\section{Acknowledgements}

628 The authors wish to acknowledge the input of Sebastian Mayer (GSK) for participant study

629 coordination advice, and Tamara Lekishvili (formerly LGC) for Flow Cytometry panel

630 advice.

631

632 Funding statement or Declaration of conflicting interests

633 This research is kindly supported by the Loughborough University Doctoral College and 634 the EPSRC/MRC Doctoral Training Centre for Regenerative Medicine at Loughborough 635 University (EP/L105072/1), GlaxoSmithKline and LGC. The authors have no conflicts of 636 interest. 


\section{References}

[1] MMIP, Advanced Therapies Manufacturing Action Plan, ABPI Taskforce Rep. (2016).

[2] I. Kite Pharma, Home | YESCARTA®, (2018). https://www.yescarta.com (accessed May 10, 2018).

[3] N. Pharmaceuticals, Kymriah(TM) Childhood Acute Lymphoblastic Leukemia Treatment, (2018). https://www.us.kymriah.com/acute-lymphoblastic-leukemiachildren/ (accessed May 10, 2018).

[4] A.P. Gee, GMP CAR-T cell production, Best Pract. Res. Clin. Haematol. 31 (2018) 126-134. doi:10.1016/j.beha.2018.01.002.

[5] H.M. Shapiro, Practical Flow Cytometry, 4th ed., John Wiley \& Sons, Hoboken, NJ, 2003.

[6] R. Vesely, J. Barths, F. Vanlangendonck, I. Hannet, K. Stmuss, Initial Results of Central European Immunophenotyping Quality Control Program ( CEQUAL ), Cytometry. 26 (1996) 108-112.

[7] M. Bergeron, S. Faucher, T. Minkus, F. Lacroix, T. Ding, S. Phaneuf, R. Somorjai, R. Summers, F. Mandy, Y. Auer, J. Luider, F. Hospital, W. Etches, S. Lee, S. Naiman, B. Waddington, S. Paul, B.G. Massing, A. Tsang, P. Keown, V. Wu, V. Hospital, B. Berry, E. Ciceri, F. Para-skevas, S. Ozamoto, M. Wilcock, B. Sahai, E. Rector, W.L. Ying, M. Macpherson, E. Zayed, H. Rankin, P. Sengar, D. Samson, O. General Hospital, L. Filion, J. Chabot, M. Richter, S. Benvie, J. Stinson, O. Civic Hospital, B. Luke, J. Chisholm, P. Pinkerton, J. Robinson, D. Pantalony, A. Chan, B. Fernandes, V. MacDonald, L. Shepherd, D. Hofmann, K. General Hospital, J. Heathcote, P. Harris, S. Joseph, Sh. Centre, I. Yee, M. Keeney, D. Snider, Impact of Unified Procedures as Implemented in the Canadian Quality Assurance Program for T Lymphocyte Subset Enumerationand the Participating Flow Cytometry Laboratories 
of the Canadian Clinical Trials Network for HIVIAIDS Therapies, Cytometry. 33 (1998) 146-155.

[8] D. Barnett, R. Louzao, P. Gambell, J. De, T. Oldaker, C.A. Hanson, Validation of

[10] J.J.M. van Dongen, A. Orfao, EuroFlow: Resetting leukemia and lymphoma immunophenotyping. Basis for companion diagnostics and personalized medicine, Leukemia. 26 (2012) 1899-1907. doi:10.1038/leu.2012.121.

[11] L. Wang, R.A. Hoffman, Standardization, calibration, and control in flow cytometry, Curr. Protoc. Cytom. 2017 (2017) 1.3.1-1.3.27. doi:10.1002/cpcy.14.

[12] ISO, BSI Standards Publication ISO 15189: Medical laboratories — Requirements for quality and competence (2014).

[13] ICH Expert Working Group, Good Manufacturing Practice Guide for Active Pharmaceutical Ingredients Q7, ICH Harmon. Trpartite Guidel. (2000) 49.

[14] N.W. Pedersen, P.A. Chandran, Y. Qian, J. Rebhahn, N.V. Petersen, M.D. Hoff, S. White, A.J. Lee, R. Stanton, C. Halgreen, K. Jakobsen, T. Mosmann, C. Gouttefangeas, C. Chan, R.H. Scheuermann, S.R. Hadrup, Automated analysis of flow cytometry data to reduce inter-lab variation in the detection of major 
histocompatibility complex multimer-binding T cells, Front. Immunol. 8 (2017) 1-12. doi:10.3389/fimmu.2017.00858.

[15] J. Spidlen, R.C. Leif, W. Moore, M. Roederer, R.R. Brinkman, Gating-ML: XMLbased gating descriptions in flow cytometry, Cytom. Part A. 73 (2008) 1151-1157. doi:10.1002/cyto.a.20637.

[19] L. Whitby, A. Whitby, M. Fletcher, M. Helbert, J.T. Reilly, D. Barnett, Comparison of methodological data measurement limits in CD4+T Iymphocyte flow cytometric enumeration and their clinical impact on HIV management, Cytom. Part B - Clin. Cytom. 84 (2013) 248-254. doi:10.1002/cyto.b.21094.

[20] J.A. Thurman-Newell, J.N. Petzing, D.J. Williams, Quantification of biological variation in blood-based therapy - a summary of a meta-analysis to inform manufacturing in the clinic, Vox Sang. 109 (2015) 394-402. doi:10.1111/vox.12288.

[21] M. Best, D. Neuhauser, Walter A Shewhart, 1924, and the Hawthorne factory, Qual. Saf. Heal. Care. 15 (2006) 142-143. doi:10.1136/qshc.2006.018093.

[22] S.L. Christensen, J.T.B. Anglov, J.M. Christensen, E. Olsen, O.M. Poulsen, Application of a new AMIQAS computer program for integrated quality control, method evaluation and proficiency testing, Anal. Bioanal. Chem. 345 (1993) 343350. 
http://dx.doi.org/10.1007/BF00043333\%5Cnhttp://springerlink.metapress.com/openu rl.asp?genre=article\&id=doi:10.1007/BF00322627.

[23] B. Brando, E. Sommaruga, Nationwide quality control trial on lymphocyte immunophenotyping and flow cytometer performance in Italy, Cytometry. 14 (1993) 294-306. doi:10.1002/cyto.990140310.

[24] D.C. Montgomery, Introduction to statistical quality control, 6th ed., Wiley, Hoboken, N.J., 2008.

[25] M.J.R. Healy, Outliers in clinical chemistry quality-control schemes, Clin. Chem. 25 (1979) 675-677.

[26] D. Barnett, L. Whitby, J. Wong, R. Louzao, J.T. Reilly, T.N. Denny, VERITAS?: A Time for VERIQAS ${ }^{\mathrm{TM}}$ and a new approach to training, education, and the quality assessment of CD4 + T lymphocyte counting (I), Cytom. Part B - Clin. Cytom. 82 B (2012) 93-100. doi:10.1002/cyto.b.20624.

[27] K.J. Goodfellow, I. Storie, V. Granger, L. Whitby, J. Antcliffe, J.T. Reilly, D. Barnett, The United Kingdom National External Quality Assessment Scheme gating and standardization strategy for use in residual WBC counting of WBC-reduced blood components., Transfusion. 42 (2002) 738-746. doi:10.1046/j.15372995.2002.00116.x.

[28] C. Leys, C. Ley, O. Klein, P. Bernard, L. Licata, Detecting outliers: Do not use standard deviation around the mean, use absolute deviation around the median, J. Exp. Soc. Psychol. 49 (2013) 764-766. doi:10.1016/j.jesp.2013.03.013.

[29] H. Paxton, P. Kidd, A. Landay, J. V Giorgi, N. Flomenberg, E. Walker, F. Valentine, J.L. Fahey, R. Gelman, Results of the flow cytometry AGTG quality control program: Analysis and findings, Clin. Immunol. Immunopathol. 52 (1989) 68-84.

[30] P. Gramatica, Principles of QSAR models validation: Internal and external, QSAR Comb. Sci. 26 (2007) 694-701. doi:10.1002/qsar.200610151. 
[31] W. Coucke, B. China, I. Delattre, Y. Lenga, M. Van Blerk, C. Van Campenhout, P. Van de Walle, K. Vernelen, A. Albert, Comparison of different approaches to evaluate External Quality Assessment Data, Clin. Chim. Acta. 413 (2012) 582-586. doi:10.1016/j.cca.2011.11.030.

[32] A. Kunkl, D. Risso, M.P. Terranova, M. Girotto, B. Brando, L. Mortara, P.B. Lantieri, Grading of laboratories on CD4+ T-lymphocyte evaluations based on acceptable data boundaries defined by the measurement error, Clin. Cytom. 50 (2002) 117126. doi:10.1002/cyto.10069.

[33] W.H.B.M. Levering, W.N. Van Wieringen, J. Kraan, W.A.M. Van Beers, K. Sintnicolaas, D.J. Van Rhenen, J.W. Gratama, Flow cytometric lymphocyte subset enumeration: 10 Years of external quality assessment in the benelux countries, Cytom. Part B - Clin. Cytom. (2008). doi:10.1002/cyto.b.20370.

[34] J. Bainbridge, W. Rountree, R. Louzao, J. Wong, L. Whitby, T.N. Denny, D. Barnett, Laboratory accuracy improvement in the uk neqas leucocyte immunophenotyping immune monitoring program: An eleven-year review via longitudinal mixed effects modeling, Cytom. Part B - Clin. Cytom. 00 (2017) 1-7. doi:10.1002/cyto.b.21531.

[35] J. Bainbridge, C.L. Wilkening, W. Rountree, R. Louzao, J. Wong, N. Perza, A. Garcia, T.N. Denny, The Immunology Quality Assessment Proficiency Testing Program for $\mathrm{CD} 3+4+$ and $\mathrm{CD} 3+8+$ lymphocyte subsets: $\mathrm{A}$ ten year review via longitudinal mixed effects modeling, J. Immunol. Methods. 409 (2014) 82-90. doi:10.1016/j.jim.2014.05.017.

[36] BD Bioscience, BD ${ }^{\mathrm{TM}}$ CS \& T Beads, (2016). http://www.bdbiosciences.com/ds/europe/tds/23-14666.pdf (accessed April 11, 2018).

[37] H.T. Maecker, J. Trotter, Flow Cytometry Controls, Instrument Setup, and the Determination of Positivity, Cytom. Part A. 69 (2006) 1037-1042. doi:10.1002/cyto.a. 
768 [38] FIRM Symposium, Previous Symposia | FIRM Symposium, (2017).

769 https://www.firmsymposium.com/copy-of-past-events (accessed April 11, 2018).

770 [39] European Commission, Good Manufacturing Practice for Advanced Therapy Medicinal Products., 4 (2017). http://ec.europa.eu/index_en.htm. 
773 List of Figures

774

775 Figure 1: Ishikawa diagram listing multiple sources of uncertainty

776

777 Figure 2: Images of histograms provided for participant gating studies (250 V to $700 \mathrm{~V}$ )

778

779 Figure 3: Comparison of inter-operator ranges of personal interpretation of histogram data

780 (left) and percentage reduction from Stage 1 to Stage 2 results (right)

781

782 Figure 4: Stain Index rCV curve

783

784 Figure 5: Application of different control limits applied to raw data

785

786 Figure 6: Application of different control limits applied to log transformed data 
790 Table 1: Common outlier estimator methods used within Flow Cytometry and other 791 industries

792

793 Table 2: Inter-operator ranges

794

795 Table 1: Summary of outlier estimator methods used to distinguish outliers from the 500 796 data set

797

798 Table 2: Control limits used to specify outliers

799 